\title{
Using strange hadrons as probes of dense matter
}

\author{
Helen Caines \\ Physics Department, Yale University, New Haven, CT 06520, U.S.A \\ E-mail: helen.caines@yale.edu
}

\begin{abstract}
.
The spectra of strange hadrons have been measured in detail as a function of centrality for a variety of collision systems and energies at RHIC. Recent results are presented and compared to those measured at the SPS. The effects of the system size on strange particle production and kinematics are examined. I place specific emphasis on comparing A-A to $p-p$ production and discuss how strangeness can be used to probe the dense matter produced in heavy-ion collisions.
\end{abstract}

\section{Introduction}

A wealth of data has and is being collected at the RHIC accelerator, BNL. The STAR experiment [1, by dint of its large acceptance for charged hadrons at mid-rapidity, is ideally suited for measuring strange hadron production. I therefore concentrate in my talk on results from STAR and contrast these to those from the SPS, CERN.

The unprecedented detail of the RHIC measurements, due to the high statistics of the data taken, allows us to probe the centrality dependence of the produced particles. The fact that RHIC can be operated over a range of collision energies and species means that the same detectors can be utilised to perform systematic scans. Since RHIC is a collider, the experiments obtain their measurements over the same phase space no matter what the collision species or energy (except for the rare case of asymmetric collisions). This, so far, unique feature of RHIC removes issues arising from differing experimental trigger and reconstruction biases when contrasting results from different experiments.

\section{Statistical models}

In ultra-relativistic heavy-ion collisions, the important question of chemical equilibration of the produced matter remains open. While there is much evidence suggesting that the matter is equilibrated, there is, as yet, no conclusive proof. The detailed study of strange hadron production, including multi-strange baryons, is key to unlocking the answer to this and other questions. One standard method for detecting chemical equilibration is via statistical models. These models aim to extract the degree of chemical equilibration of the matter, along with the freeze-out temperature, $\mathrm{T}_{c h}$. This 
is done via contrasting the experimentally measured particle ratios to those calculated from the models assuming a source in thermal equilibrium. Such models cannot prove equilibration, since the agreement may be coincidental, however if the measured ratios cannot be reproduced, the source cannot be in equilibrium at the point when the hadronic ratios are fixed.

Many such models have been applied to heavy-ion collisions, however, there are three that are readily available to experimentalists. These are, THERMUS [2], SHARE [3] and a four parameter fit model [4]. All three models have $\mathrm{T}_{c h}, \mu_{q}, \mu_{s}$ and $\gamma_{s}$ as free parameters. The latter term accounting for incomplete strangeness chemical equilibration. SHARE and THERMUS include a chemical potential to account for charge/isospin conservation, a charm chemical potential and $\gamma_{c}$. SHARE alone allows for a $\gamma_{q}$ while THERMUS can perform both Grand Canonical and Canonical ensemble calculations.

Before attempting to use these models to fit the STAR Au-Au $\sqrt{s_{N N}}=200 \mathrm{GeV}$ data using all their free parameters, a consistency check was made. This ensured that the same results were obtained when the models were constrained by a Grand Canonical calculation using only $\mathrm{T}_{c h}, \mu_{B}, \mu_{s}$ and $\gamma_{s}$. While the results were consistent within errors, a more detailed look at the models is underway in order to understand why the results are not in complete agreement.

\subsection{Fits to Central $A u$-Au and minimum bias $p$-p results.}

Figure 1a and Table 1present the results of the fits to the 0-5\% most central preliminary Au-Au data from STAR [5]. It can be seen (bottom panel of the figure) that the stable particle ratios, with the exception of the pions, are well represented by each of the models. The data for the resonances, on the other hand, are less well produced.

While each model describes the data, there are significant deviations between the results. All models calculate that the baryon and strangeness chemical potentials are close to zero yet there is a $30 \mathrm{MeV}$ difference in the chemical freeze-out temperature, this difference being several sigma beyond the models' calculated errors. THERMUS and the 4 parameter fit show strangeness saturation, $\gamma_{s}=1$, while SHARE calculates an over-saturation with $\gamma_{s}=2.1 \pm 0.6$. Within the SHARE framework, the light quarks are also over saturated with $\gamma_{q}=1.7 \pm 0.5$. The variations in these results should be taken as the degree of systematic uncertainty in the chemical freeze-out parameters determined for RHIC heavy-ion collisions. It should be noted however, that all the models determine that, when the stable particle ratios are fixed, the medium produced at RHIC is close to the critical temperature for transition to a QGP, as predicted by lattice QCD [6].

The above results should be contrasted with those of a fit to preliminary STAR

$p$ - $p$ data at $\sqrt{s_{N N}}=200 \mathrm{GeV}$ as shown in Fig. 1b. For this fit, THERMUS is used. It is currently the only readily available model that can perform a canonical ensemble fit. Again, the good quality of the fit should be noted. It is also interesting to observe 


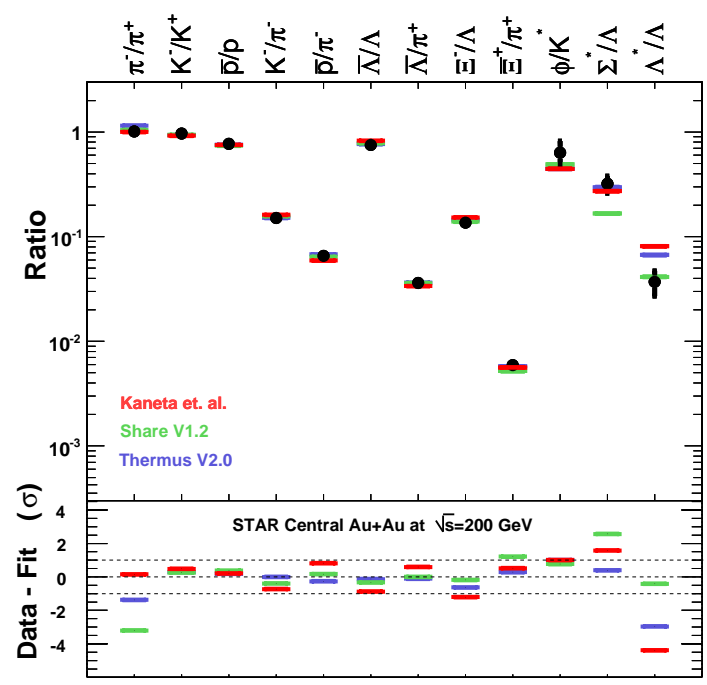

(a) Grand Canonical

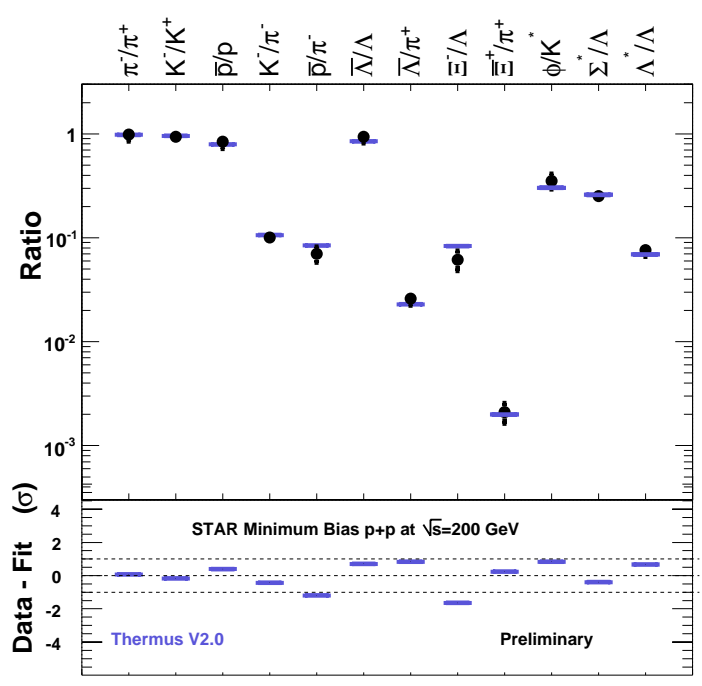

(b) Canonical

Figure 1. Statistical model fits to preliminary STAR data a) 0-5\% most central Au$\mathrm{Au}$ and b) $p-p, \sqrt{s_{N N}}=200 \mathrm{GeV}$. Solid points represent STAR preliminary data the coloured bars the calculations. The bottom panel represents the difference between the data and the calculations for each ratio.

that in the $p$ - $p$ data the resonance ratios are well represented. For this fit, $\mathrm{T}_{c h}=171$ $\mathrm{MeV} \pm 9 \mathrm{MeV}$, close to that arrived at for the Au-Au case. However, $\gamma_{s}=0.53 \pm 0.04$, indicating that the $p-p$ data are far from strangeness saturation.

\section{Centrality dependence of strangeness yields}

Since there is a marked difference in the composition of the hadrons produced in $p-p$ and central Au-Au collisions it is necessary to study the centrality dependence of strangeness production. In this way it can be determined if the transition to strangeness saturation occurs abruptly at a given centrality or changes smoothly.

\begin{tabular}{|c|c|c|c|c|c|}
\hline Model & $\mathrm{T}_{c h}(\mathrm{MeV})$ & $\mu_{B}(\mathrm{MeV})$ & $\mu_{S}(\mathrm{MeV})$ & $\gamma_{s}$ & $\gamma_{q}$ \\
\hline THERMUS & $168 \pm 6$ & $45 \pm 10$ & $22 \pm 7$ & $0.92 \pm 0.06$ & N/A \\
\hline SHARE & $133 \pm 10$ & $23 \pm 19$ & $5 \pm 7$ & $2.0 \pm 0.6$ & $1.7 \pm 0.5$ \\
\hline 4 Parameter & $161 \pm 5$ & $23 \pm 7$ & $8 \pm 5$ & $1.01 \pm 0.06$ & N/A \\
\hline
\end{tabular}

Table 1. Results from the models described in the text of Grand Canonical ensemble fits to $\mathrm{Au}-\mathrm{Au} \sqrt{s_{N N}}=200 \mathrm{GeV}$ preliminary data from STAR. 


\subsection{Strangeness enhancement}

One such study is performed using strange (anti)baryons and the enhancement factor, $\mathrm{E}(\mathrm{i})$, calculated as

$$
E(i)=\frac{\operatorname{Yield}^{A A}(i) *\left\langle N_{\text {part }}^{N N}\right\rangle}{\text { Yield }^{N N}(i) *\left\langle N_{\text {part }}^{A A}\right\rangle} .
$$

If the yields of the hyperons scale with $\left\langle N_{\text {part }}\right\rangle, \mathrm{E}(\mathrm{i})$ will be unity over all centralities. Such a scaling is predicted in the Grand Canonical regime when strangeness is fully equilibrated.

Figure 2a shows the results of this calculation at RHIC [7] compared to those from NA57 at the SPS for Pb-Pb collisions at $\sqrt{s_{N N}}=17.3 \mathrm{GeV}$ [, 9]. Also shown are the results for STAR's measured inclusive protons, which include all feed-down protons from hyperon decays. It can be seen that the enhancement increases with increasing strangeness content and that, at RHIC, the anti-baryon enhancements are approximately those of the baryons. This reflects the near zero net-baryon density at RHIC. While the enhancements are similar between RHIC and SPS data, the magnitude of the normalization uncertainty, reflected by the error boxes at $\left\langle N_{\text {part }}\right\rangle=1$ should be noted. This normalization uncertainty is due to the error in the $p$ - $p$ and $\mathrm{p}$-Be data and can only be reduced by improving the event statistics for these collisions.

The solid boxes on the right hand axis of Fig. 2a represent the predicted enhancements using a statistical model calculation. This model uses a canonical regime calculation for $p-p$ and assumes that the Grand Canonical regime is reached in central AA collisions [10]. The bottom edge of the boxes uses a chemical freeze-out temperature, $\mathrm{T}_{c h}$, of $170 \mathrm{MeV}$ for both $p-p$ and $\mathrm{Au}-\mathrm{Au}$ collisions. The top of the boxes represents the same model calculation for $\mathrm{T}_{c h}=165 \mathrm{MeV}$. This range of temperatures is within the uncertainty of the chemical freeze-out temperature obtained in these collisions, as discussed in section 2. The predicted enhancement decreases with increasing freeze-out temperature since the enhancement within this model is due to reduced phase space for strangeness production in $p$ - $p$. The available phase space increases with temperature and volume of the produced fireball and hence the enhancement decreases if either term increases. It appears from this calculation that the model cannot differentiate between the two temperatures.

However, as stated, the enhancement is sensitive to both the temperature of the system and its volume. This volume term is proportional to the cube of the proton radius, $R_{0}$. This cubic dependency implies that the enhancement factors are very sensitive to the assumed radius, Fig. 2b [11]. This dual dependency on $\mathrm{T}_{c h}$ and $\mathrm{R}_{0}$ means that a given enhancement can be obtained via two combinations, the multistrange baryons having the greatest sensitivity.

It is believed that there is a linear correlation between the geometric overlap volume of the collision and $\left\langle N_{\text {part }}\right\rangle$. The strangeness production correlation volume and $\left\langle N_{\text {part }}\right\rangle$ were also expected to be linearly related. However, when a comparison of the shape of the enhancement as a function of $\left\langle N_{\text {part }}\right\rangle$ to that predicted by the model assuming a 


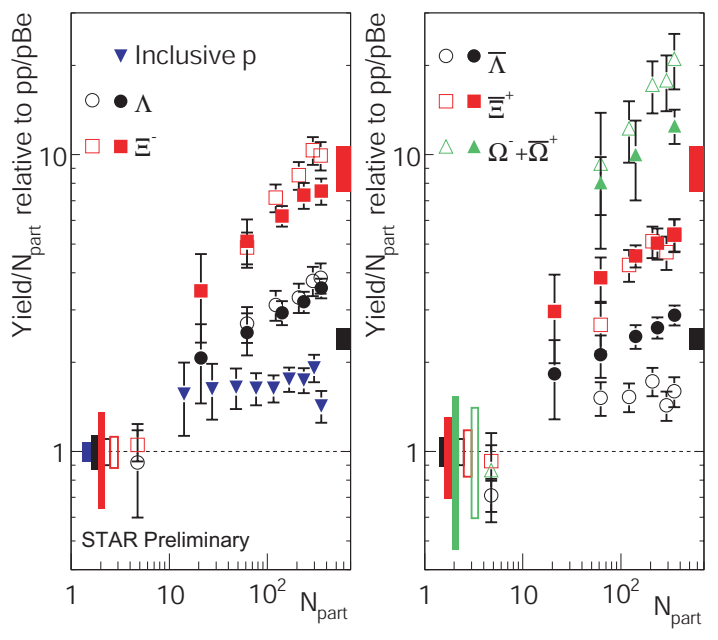

(a)

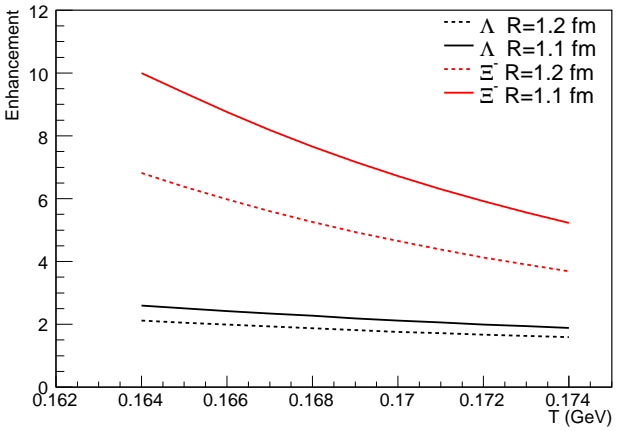

(b)

Figure 2. a) Enhancement factors as a function of $\left\langle N_{\text {part }}\right\rangle$ for strange (anti)baryons. Solid symbols are for $\mathrm{Au}-\mathrm{Au}$ collisions at $\sqrt{s_{N N}}=200 \mathrm{GeV}$, and the open symbols are measured by NA57 from $\mathrm{Pb}-\mathrm{Pb}$ at $\sqrt{s_{N N}}=17.3 \mathrm{GeV}$. The boxes represent the combined statistical and systematical uncertainties in the $p$ - $p$ and p-Be data. The error bars on the data points represent those from the heavy-ion measurement. The boxes on the right axes mark the predictions from a model using a Grand Canonical formalism. b) Calculated enhancements using the model described in 10 as a function of chemical freeze-out temperature. Two values of $R_{0}$ are assumed to calculate the strangeness production correlation volume.

linear term in $\left\langle N_{\text {part }}\right\rangle$ is made (see the solid curve in Fig. 3a) the measured functional form is not well represented. Figure [3a also shows a $\left\langle N_{\text {part }}\right\rangle^{2 / 3}$, dashed curve, and $\left\langle N_{\text {part }}\right\rangle^{1 / 3}$, dashed-dot curve. The best description of the data, in both shape and magnitude, is achieved when a $\left\langle N_{\text {part }}\right\rangle^{1 / 3}$ scaling is used in combination with $\mathrm{T}_{c h}=165$ $\mathrm{MeV}$ and a radius $\mathrm{R}_{0}=1.1 \mathrm{fm}$.

\subsection{Flavour dependence of the scaled yields}

When studying the enhancement for strange baryons it is helpful to also examine the possible enhancement of non-strange baryons and mesons. Figure 3 b shows the results for identified $\pi^{-}, \mathrm{K}^{-}$and inclusive, i.e. including all feed-down, anti-protons 12 ] compared to $\bar{\Lambda}$ at $\sqrt{s_{N N}}=200 \mathrm{GeV}$. It can be seen that there is an enhancement for all species and that the enhancement scales with the number of (anti-)strange quarks in the particle. While it is expected that the strange quarks "see" a phase space suppression in peripheral A-A and $p$ - $p$ collisions the light quarks are not expected to be affected in such a manner. Since the models do not predict an enhancement for anti-protons and pions, any phase-space suppression effects for strangeness should be made relative to the observed increase in the measured pion yields. 


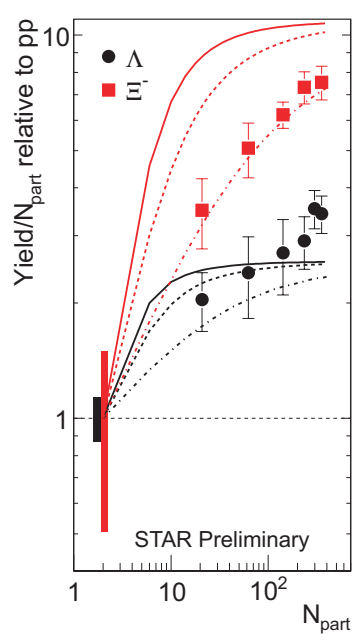

(a)

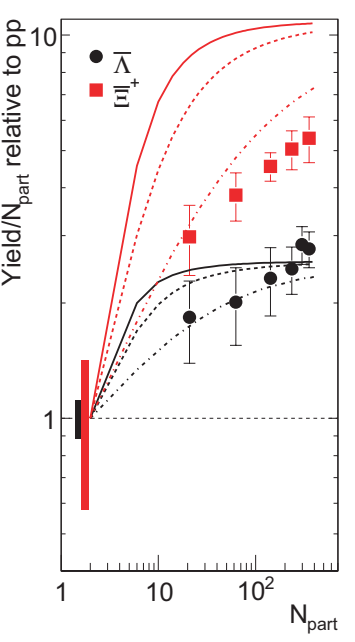

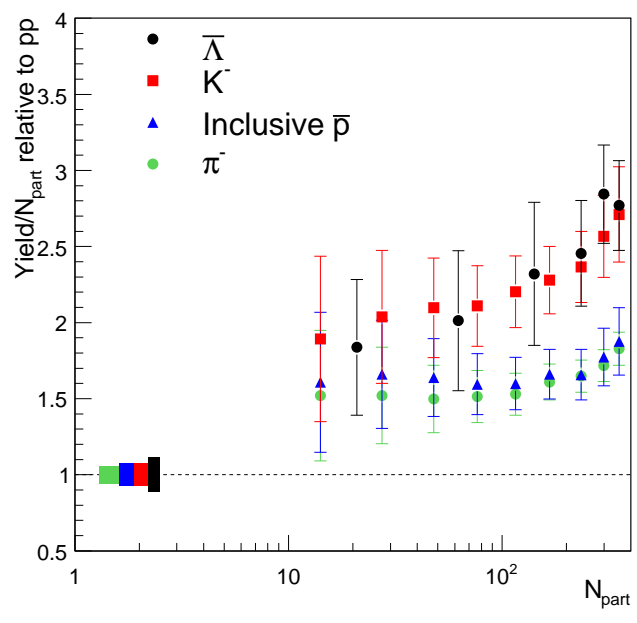

(b)

Figure 3. a) Enhancement factors as a function of $\left\langle N_{\text {part }}\right\rangle$ for strange (anti)baryons. Errors as explained in Fig. (1/n). The curves are for different assumptions of the $\left\langle N_{\text {part }}\right\rangle$ dependence of the strangeness suppression. The solid curve is $\left\langle N_{\text {part }}\right\rangle$ scaling, dashed curve $-\left\langle N_{\text {part }}\right\rangle^{2 / 3}$, dot-dashed curve $-\left\langle N_{\text {part }}\right\rangle^{1 / 3}$. A common $\mathrm{T}_{c h}$ of $165 \mathrm{MeV}$ is assumed. b) The enhancements factor calculated for preliminary data from STAR for identified particles. The data are from STAR for Au-Au collisions at $\sqrt{s_{N N}}=200 \mathrm{GeV}$.

\section{Beyond the bulk}

So far I have dealt only with integrated yields, a measure dominated by the low $\mathrm{p}_{T}$ region, $\mathrm{p}_{T}<1 \mathrm{GeV} / \mathrm{c}$. To probe the particle production further, we study the nuclear modification factor, $\mathrm{R}_{A A}$. The nuclear modification factor compares $\mathrm{p}_{T}$ spectra from A-A collisions with binary scaled $p$ - $p$ data. When $p$ - $p$ results are not available an alternative measure, $\mathrm{R}_{C P}$, is calculated by substituting binary scaled peripheral A-A results for the $p$ - $p$ spectra. When using $\mathrm{R}_{C P}$, it is assumed that the peripheral A-A data are a close approximation to $p$ - $p$ at the same energy. However, in the previous sections I have shown that the $\left\langle N_{\text {part }}\right\rangle$ scaled central yields of $\Lambda$ and $\Xi$ are enhanced by factors of 3 and 7 respectively, compared to $p$ - $p$. Even the most peripheral $\left\langle N_{\text {part }}\right\rangle$ scaled data are a factor of 2-3 enhanced. Thus, the assumption that peripheral data can be substituted for $p$ - $p$ is clearly incorrect. However, a study of both measures remains informative.

The measured $\mathrm{R}_{A A}$ for $0-5 \% \mathrm{Au}-\mathrm{Au}$ for $\Lambda, \Xi$ and inclusive protons has been shown previously [13]. This data show that $R_{A A}(\Xi)>R_{A A}(\Lambda)>R_{A A}(\overline{\mathrm{p}})>R_{A A}\left(K_{S}^{0}\right)$ at intermediate $\mathrm{p}_{T}$. All particles except the kaon peak above unity. The $\mathrm{R}_{C P}$ measure, on the other hand, shown in Fig. 4a, shows $\mathrm{R}_{A A}(\Xi)=\mathrm{R}_{A A}(\Lambda)=\mathrm{R}_{A A}(\overline{\mathrm{p}})$ and an $\mathrm{R}_{C P}$ maximum of approximately 0.8 [7]. The inclusion of the kaon and $\pi$ in Fig. 4a also reveals a baryon/meson splitting up to $\mathrm{p}_{T}$ values of $\sim 6 \mathrm{GeV} / \mathrm{c}$, after which a constant 
suppression at 0.2 is observable for all species. This suppression with respect to binary scaling is taken as evidence of energy loss of partons as they pass through the hot medium produced in central Au-Au collisions at RHIC [14, 15. Meanwhile the baryon/meson splitting at intermediate $\mathrm{p}_{T}$ can be explained via quark recombination models [16]. The observed suppression of high $\mathrm{p}_{T}$ particles in central $\mathrm{Au}-\mathrm{Au}$ events and the baryon/meson splitting at intermediate $\mathrm{p}_{T}$ are strong indications that the system enters a partonic phase with a large gluon density. The striking difference between the $\mathrm{R}_{C P}$ and $\mathrm{R}_{A A}$ measurements can be understood in terms of the large phase space suppression effects, which dominate the bulk measurements, extending into the high $\mathrm{p}_{T}$ domain. The $\mathrm{R}_{A A}$ is therefore a convolution of two effects with the phase space suppression dominating. However, in peripheral events, the phase space effects are already significantly diluted but those of the hot dense medium are not yet large. $\mathrm{R}_{C P}$, for strange particles, is therefore a more sensitive measure of "jet quenching" effects at intermediate and high $\mathrm{p}_{T}$.

$\mathrm{R}_{C P}$ measurements have also been made in $\mathrm{Au}-\mathrm{Au}$ collisions at $\sqrt{s_{N N}}=62 \mathrm{GeV}$ at RHIC [13] and at the SPS for $\sqrt{s_{N N}}=17.3 \mathrm{GeV}$ [17. In both cases, there is a clear splitting between the $\Lambda$ measurements and the $\mathrm{K}_{S}^{0}$ measurements in the intermediate $\mathrm{p}_{T}$ regime. While the peaks of all the measurements occurs at approximately the same $\mathrm{p}_{T}$ for all three measurements the value of the maxima increases as the collision energy decreases. The peak of the SPS measurements have a value of $\sim 1.3$ and 1 for $\Lambda$ and $\mathrm{K}_{S}^{0}$ respectively while the top RHIC values are $\sim 0.9$ and 0.7 . The splitting of the baryons and mesons however appears to be similar. To probe this probability further, we plot in Fig. 4b the $\mathrm{R}_{C P}(\Lambda) / \mathrm{R}_{C P}\left(K_{s}^{0}\right)$ ratio. It is striking that the shape of this ratio is the same for each of the three energies. This suggests that any conclusions drawn from the splitting of baryons/mesons at $\sqrt{s_{N N}}=200 \mathrm{GeV}$ should also be concluded for top SPS energies. It may however be accidental but that would require three different sets of mechanisms happening to sum to the same result which is unlikely.

\section{Summary}

In summary, in central Au-Au collisions at energies of $\sqrt{s_{N N}}=200 \mathrm{GeV}$, we observe a distinct enhancement of strangeness production compared to that in $p$ - $p$ collisions at the same energy. If the origin of the measured enhancement is due to a lack of available phase space in $p$ - $p$ and peripheral collisions, then these phase space effects diminish with an apparent $\left\langle N_{\text {part }}\right\rangle^{1 / 3}$ dependency. The effects of this enhancement can be observed into the intermediate $\mathrm{p}_{T}$ regime.

A clear suppression of intermediate and high $\mathrm{p}_{T}$ particles is observed. It can be explained via models assuming partonic energy loss. Phase space suppression effects make the $\mathrm{R}_{C P}$ ratio a cleaner measurement from which to extract "jet quenching" effects since $\mathrm{R}_{A A}$ is a convolution of phase space suppression and partonic energy loss effects. The baryon/meson splitting at intermediate $\mathrm{p}_{T}$ is seen at all energies from $\sqrt{s_{N N}}=17.3$ to $200 \mathrm{GeV}$. Although the scale of the suppression is reduced at lower energies the slope 


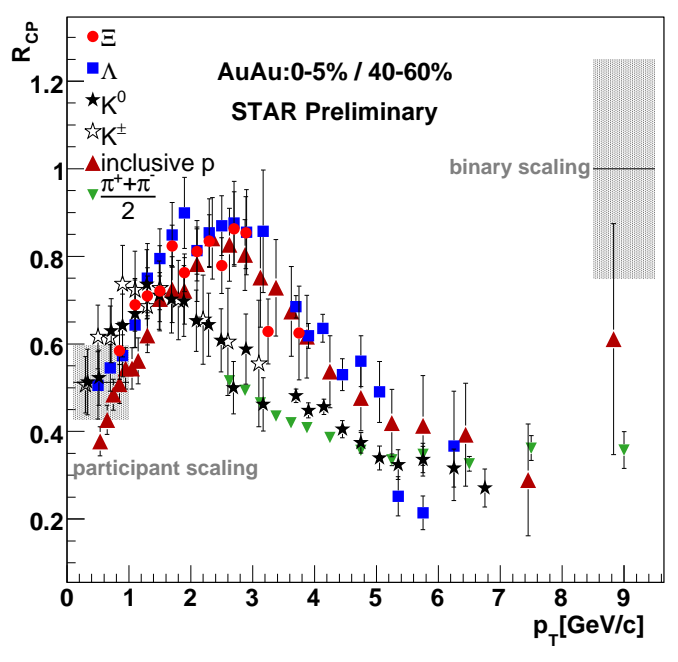

(a) $\mathrm{R}_{C P}$ for identified particles.

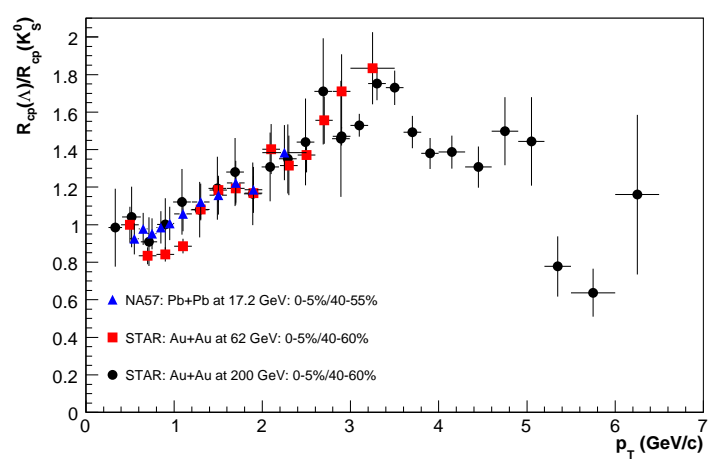

(b) $\mathrm{R}_{C P}(\Lambda) / R_{C P}\left(K_{S}^{0}\right)$.

Figure 4. a) $\mathrm{R}_{C P}$ for $\mathrm{Au}-\mathrm{Au}$ collisions at $\sqrt{s_{N N}}=200 \mathrm{GeV}$. b) The ratio of the $\mathrm{R}_{C P}(\Lambda)$ over $\mathrm{R}_{C P}\left(K_{s}^{0}\right)$ for different collisions energies from RHIC and the SPS.

of fractional difference between the $\Lambda$ and $\mathrm{K}_{S}^{0} \mathrm{R}_{C P}$ values is the same. This suggests that recombination is present at both RHIC and top SPS energies.

\section{Acknowledgments}

I wish to thank Krzysztof Redlich for very helpful discussions and providing many of the theoretical curves. Thanks also go to Sevil Salur, Georgio Torrieri, Ingrid Kraus, and Anton Andronic for working with me to investigate the various statistical models and their differences.

\section{References}

[1] M. Anderson et al. (STAR Collaboration), Nucl. Instrum. Meth. A 499, 659 (2003).

[2] S. Wheaton and J. Cleymans, hep-ph/0407174 (2004).

[3] G. Torrieri et al., Comput. Phys. Commun 167229 (2005).

[4] N. Xu and M. Kaneta, Nucl. Phys. A698 306c (2002).

[5] S. Salur, (for the STAR Collaboration) nucl-ex/0606006 (2006).

[6] F. Karsch J. Phys. G 31641 (2005).

[7] M.A.C. Lamont (for the STAR Collaboration), these proceedings.

[8] E. Andersen et al. (WA97 Collaboration), Phys. Lett. 449401 (1999).

[9] F. Antinori (for the WA97/NA57 Collaboration), Nucl. Physics A698 118 (2002).

[10] A. Tounsi, A. Mischke and K. Redlich, Nucl. Phys. A715 565 (2003).

[11] K. Redlich private communication, based on method described in [10.

[12] J. Adams et al. (STAR Collaboration), Phys. Rev. Lett. 92112301 (2004).

[13] S. Salur, (for the STAR Collaboration) QM2005 nucl-ex/0509036 (2005).

[14] M. Gyulassy and M. Plumer, Phys. Lett. B 243432 (1990); R. Baier et al. ibid 345, 277 (1995). 
[15] X.N. Wang and M. Gyulassy, Phys. Rev. Lett. 681480 (1992); X.N. Wang Phys. Rev. C 58, 2321 (1998).

[16] R.J. Fries et al. Phys. Rev. C 68044902 (2003), V. Greco et al. Phys. Rev. C 68034904 (2003) and R.C. Hwa et al. Phys. Rev. C 67034902 (2003).

[17] F. Antinori et al. (NA57 Collaboration) Phys. Lett. B 62317 (2005). 